\title{
Improving Maladaptive Behavior: The Effect of Episodic Foresight on Delay Discounting and Its Mechanism
}

\author{
Wenwen Yang, Yaozhong Liu \\ School of Management, Jinan University, Guangzhou, China \\ Email: yangwenwen12@qq.com
}

How to cite this paper: Yang, W. W., \& Liu, Y. Z. (2019). Improving Maladaptive Behavior: The Effect of Episodic Foresight on Delay Discounting and Its Mechanism. Psychology, 10, 19-29.

https://doi.org/10.4236/psych.2019.101002

Received: December 11, 2018

Accepted: January 7, 2019

Published: January 10, 2019

Copyright (๑) 2019 by author(s) and Scientific Research Publishing Inc. This work is licensed under the Creative Commons Attribution International License (CC BY 4.0).

http://creativecommons.org/licenses/by/4.0/

\section{(c) (i) Open Access}

\begin{abstract}
Delay discounting is an important part of intertemporal decision-making. This decision involves information about the costs and benefits of future possibilities, which affect people making decision. Episodic foresight therefore has a capacity to organize current action in view of anticipated events and concerns the imaginative of thinking about future events that often draw on the visual modality. In the clinic research, episodic foresight could improve maladaptive behaviors, including pathological gambling, obesity, drug and alcohol abuse. Our study explores the psychological mechanism and brain mechanism. We proposed that future research should further explore how the episodic foresight affects delay discounting and how episodic foresight is applied to clinic and behaviour modification.
\end{abstract}

\section{Keywords}

Episodic Foresight, Delay Discounting, Maladaptive Behavior, Mechanism

\section{Introduction}

Lots of studies have shown that it is impossible to suppress their own impulse preferences, and delaying satisfaction is a hallmark of many maladaptive behaviors, such as pathological gambling, overeating, alcohol and drugs abuse (Bickel \& Marsch, 2001; Epstein et al., 2010; Mackillop et al., 2010; Odlaug, Schreiber, \& Grant, 2013). At present, extensive research uses the effects of delay discounting (smaller instant rewards and larger reward preferences) to study impulsive behavior, which is considered an impulsive behavioral indicator (Ainslie, 1975).

Delay discounting is an important part of intertemporal decision-making. Intertemporal decision-making is a future-oriented decision-making mechanism 
that contains information about costs and benefits of future possibilities. Humans can make mental time travel or episodic foresight for the future. This ability allows people to imagine potential future rewards and their possible scenarios, thus providing information for decision making (Atance \& O'Neill, 2001; Suddendorf \& Corballis, 2010; Suddendorf \& Moore, 2011). Simulating future rewards in an intertemporal decision-making scenario may lead people to abandon immediate enjoyment and pursue longer-term goals (Boyer, 2008). Therefore, it can be considered that the scenario expectation can effectively reduce the delay discounting and enhance people's self-control level. This study first sorts out and summarizes relevant literature and internal mechanisms such as scenario expectations and delay discountings. Then, from the aspects of obesity, gambling, alcohol abuse and tobacco and drug abuse caused by poor diet, the scenario expectation can control the impulse and improve the maladaptive behavior. Finally, it further analyzes the influencing factors of the episodic foresight on the delay discounting and forecasts the future research direction.

\section{Episodic Foresight Improving Maladaptive Behavior}

Although individuals may not explicitly determine the benefits at different times when they choose whether to eat unhealthy food or drink, the intertemporal decision-making process is common to the processes used in this health-related consumption decision (Yi, Mitchell, \& Bickel, 2010). In this case, people have such choices: 1) prioritize decisions that satisfy immediate (e.g. happy), or 2) prioritize decisions about long-term factors (e.g. health).

\subsection{Episodic Foresight Reduce Impulsive Diet}

Obesity seems to be related to people's preference for immediate rewards. The taste of high-sugar and high-fat foods is popular, but we must weigh instant happiness and long-term health effects (such as healthy weight and health risks) (Hall \& Fong, 2007). Recent studies have found that impulses are significantly associated with food addiction. 1) Positive urgency and negative urgency are mixed together, and people show impulsive responses in a strong emotional state. 2) Food addicts shows a steep delay discounting rate. The results also demonstrate that food addiction is an intermediary between urgency and delay discounting for obese people. These findings provide further evidence linking impulsiveness to food addiction and obesity, and suggest that food addiction may be one of the causes of obesity in individuals (Vanderbroek-Stice, Stojek, Beach, Vandellen, \& Mackillop, 2017).

Some studies used the EFT/EPT paradigms, that is, let one group of subjects imagine the future situation, and the other group imagine the recent events to study the calorie intake of obese women and overweight children during the free diet. The results showed that the imagination can indeed control the impulse diet in the future (Daniel, Said, Stanton, \& Epstein, 2015; Daniel, Stanton, \& Epstein, 2013). However, Daniel, Stanton, \& Epstein (2013) allowed the participants 
to imagine positive future events, but the content of the images was not limited. Therefore, Dassen, Jansen, Nederkoorn and Houben (2016) recently explored whether food-related EFT is more effective in promoting healthy dietary choices. It turns out that EFT does reduce the delay discounting, but this effect is not determined by the content: in the EFT group, the delay discounting is reduced compared to the general food-related imagination and control group. In addition, they also found that only food-related events are expected to reduce food intake. Specifically, future imaginations reduce impulse decisions, and only food-related imaginations affect calorie intake. Future research should continue to focus on the role of episodic foresight in the adjuvant treatment of unhealthy diets such as bulimia.

\subsection{Episodic Foresight Reduce Gambling Behavior}

Pathological gambling has recently been defined as an addictive behavior disorder, and many studies have now shown an increase in delay discounting (Miedl, Peters, \& Büchel, 2012; Wiehler, Bromberg, \& Peters, 2015). Executive control is considered the key to resisting attractive, instant rewards. Therefore, the implementation of control disorders may be one of the reasons for the steep delay discounting rate in Pathological Gambling (PG). Studies have shown that executive control of pathological gambling in the Stroop task is impaired and is associated with changes in the medial prefrontal cortex of the left ventral (Potenza, 2003). The results showed that the executive control of the PG group may be impaired compared to the healthy subjects in the control group. At the same time, some researchers believe that the future-oriented thinking ability of pathological gambling patients will be reduced. Specifically, they think of fewer future events in future event tasks, reduced forward thinking, lack of contextual detail and richness (sensory details and contextual information), and are associated with reduced self-awareness (Noël, Saeremans, Kornreich, Jaafari, \& D'Argembeau, 2017). Less contact with future thinking may also lead to an increase in the delay discounting rate.

All in all, the above studies suggest that gambling addiction is associated with a decline in the ability to lead thinking in the future, especially in the context of a foresight. In the future, we should further explore the structure and process of the future thinking of pathological gamblers, which may innovate the form of treatment.

\subsection{Episodic Foresight Reduce Alcohol Abuse}

Studies have shown that the delay discounting for subjects who abuse alcohol is significantly higher than that of the control group (Bjork, Hommer, Grant, \& Danube, 2004; Field, Christiansen, Cole, \& Goudie, 2007). This indicates that the participants' substantive decision-making bias for immediate rewards is at the expense of future benefits, which is the basis of the impulsive behavior of alcohol abusers and dependents (i.e. unable to adhere to controlled drinking or give up 
when they can drink immediately) (Vuchinich \& Heather, 2003). Further evidence suggests that alcohol abusers may not be able to effectively imagine and plan for the future, as their prospective memory and ability to plan for the future have been shown to be inversely related to their degree of alcohol dependence (Griffiths et al., 2012). Then, we can think that alcohol addicts can't reasonably expect future rewards, resulting in overestimation of instant rewards and higher delay discountings.

Snider, Laconte and Bickel (2016) argued that episodic foresight may expand an individual's temporal window and then make higher valuations and more self-control for the future. They randomly assigned alcohol-dependent subjects to the EFT group and the control group, and asked for a positive future or near-term event for each of the five time points, followed by a delayed discount task, during which event prompts were displayed, and Hypothetical alcohol purchase mission. It was found that the EFT group significantly increased the valuation of future rewards, while the initial consumption of alcoholic beverages decreased, indicating a decrease in the intensity of alcohol demand. Similarly, Bulley and Gullo (2017) used college students as subjects, modified intertemporal selection paradigms and hypothetical shopping tasks, during which subjects were shown personally relevant future event reminders or control image prompts. The results were consistent with the predecessors and found that the delays in the expected group of participation scenarios were significantly reduced, and the initial intensity of alcohol demand (rather than other demand indices) was also lower relative to the control group.

In summary, episodic foresight can reduce delay discounting while also predicting the intensity of alcohol demand. Relevant researchers and practitioners can develop novel and effective interventions and correction strategies for behavioral disorders characterized by immediate rewards for ignoring long-term rewards.

\subsection{Episodic Foresight Reduce Tobacco and Drug Abuse}

The depreciation of delay discounting or delayed rewards is an effective indicator of the use of tobacco and other drugs (Bickel, Koffarnus, Moody, \& Wilson, 2014). Tobacco and drug addicts and rapid devaluation of future (e.g. long-term good health) titers may help increase the relative value of immediate drug action. Recent investigators selected heroin addiction patients and found that the patient group showed a higher rate of delayed discount compared to the control group. Further analysis suggests that this is because the patient group reduces sensitivity to optimal decision making, which is reflected in lower information accumulation rates and higher decision criteria (Scherbaum, Haber, Morley, Underhill, \& Moustafa, 2017).

Stein et al. (2016) studied the effects of episodic foresight on delay discounting and tobacco self-management in smokers. The study used the EFT/ERT paradigms and ensured that the contextual content in both groups was related to 
memory and was autobiographical. It was found that EFT can reduce the delayed discount for smokers compared to ERT. Researchers believe that episodic foresight shifts the time perspective and leads to improvements in the valuation of current and future consequences, increasing the likelihood of considering long-term benefits, such as decisions about whether to smoke.

\section{The Mechanism of Episodic Foresight in Delay Discounting}

\subsection{Psychological Mechanisms}

Imagining future events is a skill that allows us to put ourselves in the future through psychological simulations, which increases self-association with the future and activates forward-thinking thinking (Hershfield, 2011). At the same time, an ideal self may lead to a future-oriented mindset that promotes future considerations and reduces delay discounting (Cheng, Shein, \& Chiou, 2012). Recently, Wu, Cheng and Chiou (2017) let the subjects imagine future events related to the self. They found that imagining the future ideal self can trigger a lower discount rate, and by considering the cost of the result, thereby increasing the crimes and Cheating resistance. The Construal Level Theory (CLT) argues that in the process of individual characterization of delayed rewards, money is at a high level of interpretation, while latency is at a low level of interpretation (Liberman \& Trope, 2003). That is to say, if the subjects give more weight to the time that belongs to the low-level explanation, and therefore prefer to make short-term choices, they will show a larger delay discounting. However, events that are relatively distant in time will provoke a high level of interpretation. People will tend to be high-interpretation levels rather than low-interpretation levels. High-level behavioral signs represent more abstract behaviors; advanced description (Trope \& Liberman, 2000). Therefore, the scenario is expected to shift people's focus to the future and lead to future-oriented thinking. This makes individuals tend to interpret at a high level, give greater weight to money, tend to choose delayed rewards, and reduce delay discounting.

Liu, Feng, Chen, and Li (2013) argued that emotions have a regulatory effect on the effects of scenario expectations on delayed discounts. They allow participants to imagine positive future events, negative future events, and neutral future events. As a result, it was found that compared with the imaginary future subjects, the participants who imagined the positive events in the future prefer to delay the reward, and the subjects who imagine the negative future events prefer the immediate reward. Therefore, it can be assumed that episodic foresight may influence an individual's decision-making through positive or negative emotions. This is consistent with the emotion-of-episodic-prospection hypothesis, which assumes that imagining the future can trigger a specific mental time travel experience, which does not require deliberate extraction or construction. Once triggered, they usually activate the pathway of emotions, and emotions affect how people feel about the subjective value of the options. These emotions do not require cognitive control and are spontaneous. In other words, imagination can 
play a role in reducing or increasing patience by linking our choices to positive or negative uncontrolled emotions (Liu et al., 2013).

\subsection{Brain Mechanisms}

Peters and Büchel (2011) used fMRI to find three major neural networks for delay discountings, reward valuation, cognitive control, and scenario prediction. Value assessment includes the ventral medial prefrontal cortex (vmPFC), medial cortex prefrontal cortex (mOFC), the posterior cingulate cortex (PCC), representing the decision maker's subjective discount on future reward values. Cognitive control consists of lateral prefrontal cortex (lateral PFC) and anterior cingulate cortex (ACC), which is responsible for monitoring conflicts between options and adjusting the selection strategy. The episodic foresight includes the medial temporal lobe region (MTL) of the hippocampus, which is determined by characterizing future potential possibilities. At the same time, the stronger the functional connection between the current buckle belt and the hippocampus and the amygdala, the less impulsive decisions were made (Peters \& Büchel, 2010). In short, when people imagine the future events, the hippocampus area is activated, and the activation of the hippocampus transmits information to the prefrontal cortex, specifically the anterior cingulate cortex, which leads to enhanced activation of the anterior cingulate cortex, while the anterior cingulated. The enhancement of cortical activation is positively correlated with cognitive control, which makes people's subjective value for future rewards higher and the impulsiveness of subjects reduced (Wang \& Zhang, 2015).

The medial temporal lobe (MTL) plays an important role in imagining future scenarios when making decisions. This kind of imagining future effect allows people to reduce delay discounting. Palombo, Keane and Verfaellie (2015) have recently discovered that in patients with amnesia who are impaired in MTL, the delayed discount after imagining the future is different from that of normal subjects. Specifically, patients with amnesia are imagining a specific future event; it is not possible to choose an option that requires more patience. More and more studies have shown that hippocampal damage not only damages episodic memory, but also impairs the expected function of the scenario (Kwan, Carson, \& Rosenbaum, 2010; Kwan, Craver, Green, Myerson, \& Rosenbaum, 2013). Delay discounting in hippocampal-injured mice are increased (Mariano et al., 2009), and there is also a decline in the ability to investigate novel events in hippocampal lesions (Hassabis, Kumaran, Vann, \& Maguire, 2007). Current research suggests that hippocampus and hippocampal cortex play a key role in characterizing vivid events, whether they occur in the past, present or future (Schacter \& Addis, 2009). The hippocampus can project self-projection into the future to facilitate decision making (Schacter, Addis, \& Buckner, 2007), allowing people to assess future returns through psychological simulations (Johnson \& Redish, 2007).

\section{Future Research}

In summary, episodic foresight can reduce delay discounting and can effectively 
improve maladaptive behaviors such as pathological gambling, obesity, alcohol and drug abuse, and the like. An understanding of the impact of episodic foresight on delay discounting can help us make more effective and informed decisions in our lives, as well as a means of preventing and improving maladaptive behavior. However, in a strict scientific sense, this issue needs to be strengthened in the following aspects.

First, future research should continue to delve into the mechanisms by which episodic foresight affect intertemporal decision making. The literature has shown that non-human animals and people with hippocampal amnesia, although their scenarios are expected to be functionally or severely restricted, can at least select a longer delay reward in a short period of time (Kwan et al., 2012; Stevens \& Stephens, 2008). At the same time, episodic foresight is not only reducing delay discountings. When exposed to a thread with a risk of death, the act of choosing instant rewards may instead be an adaptive strategy. Therefore, under these conditions, people are more likely to receive instant rewards (Hill, Jenkins, \& Farmer, 2008; Pepper \& Nettle, 2013, 2014), with a delay in discounts. In short, episodic foresight enable humans to take into account possible scenarios at different points in time and their connections to current decisions, so that people can make choices cautiously. The adaptive function of the episodic foresight is not only a far-sighted pursuit of future big rewards, but also an immediate reward when the expected content is severe (Bulley, Henry, \& Suddendorf, 2016).

Second, future research should continue to focus on the role of episodic foresight to improve bad behavior, and researchers can then target forward-thinking imaginative intervention strategies for bad behavior. At the same time, we also note that the way to improve maladaptive behavior by reducing the ability to delay discounting is not limited to episodic foresight. Other experimental variables known to reduce discounting, such as mindfulness and basic governance (Hendrickson \& Rasmussen, 2013), explicit training can also increase resistance to delayed discounts (Stein, Renda, Hinnenkamp, \& Madden, 2015) or work Memory training (Bickel, Yi, Landes, Hill, \& Baxter, 2011) may also help to reduce subsequent drug use by extending the perception of time to time.

\section{Conflicts of Interest}

The authors declare no conflicts of interest regarding the publication of this paper.

\section{References}

Ainslie, G. (1975). Specious Reward: A Behavioral Theory of Impulsiveness and Impulse control. Psychological Bulletin, 82, 463-496. https://doi.org/10.1037/h0076860

Atance, C. M., \& O’Neill, D. K. (2001). Episodic Future Thinking. Trends in Cognitive Sciences, 5, 533-539. https://doi.org/10.1016/S1364-6613(00)01804-0

Bickel, W. K., \& Marsch, L. A. (2001). Toward a Behavioral Economic Understanding of Drug Dependence: Delay Discounting Processes. Addiction, 96, 73-86. 
https://doi.org/10.1046/j.1360-0443.2001.961736.x

Bickel, W. K., Koffarnus, M. N., Moody, L., \& Wilson, A. G. (2014). The Behavioral- and Neuro-Economic Process of Temporal Discounting: A Candidate Behavioral Marker of Addiction. Neuropharmacology, 76, 518-527. https://doi.org/10.1016/j.neuropharm.2013.06.013

Bickel, W. K., Yi, R., Landes, R. D., Hill, P. F., \& Baxter, C. (2011). Remember the Future: Working Memory Training Decreases Delay Discounting among Stimulant Addicts. Biological Psychiatry, 69, 260-265. https://doi.org/10.1016/j.biopsych.2010.08.017

Bjork, J. M., Hommer, D. W., Grant, S. J., \& Danube, C. (2004). Impulsivity in Abstinent Alcohol-Dependent Patients: Relation to Control Subjects and Type 1-/Type 2-Like Traits. Alcohol, 34, 133-150. https://doi.org/10.1016/j.alcohol.2004.06.012

Boyer, P. (2008). Evolutionary Economics of Mental Time Travel? Trends in Cognitive Sciences, 12, 219-224. https://doi.org/10.1016/j.tics.2008.03.003

Bulley, A., \& Gullo, M. J. (2017). The Influence of Episodic Foresight on Delay Discounting and Demand for Alcohol. Addictive Behaviors, 66, 1-6.

https://doi.org/10.1016/j.addbeh.2016.11.003

Bulley, A., Henry, J., \& Suddendorf, T. (2016). Prospection and the Present Moment: The Role of Episodic Foresight in Intertemporal Choices between Immediate and Delayed Rewards. Review of General Psychology, 20, 29-47. https://doi.org/10.1037/gpr0000061

Cheng, Y. Y., Shein, P. P., \& Chiou, W. B. (2012). Escaping the Impulse to Immediate Gratification: The Prospect Concept Promotes a Future-Oriented Mindset, Prompting an Inclination towards Delayed Gratification. British Journal of Psychology, 103, 129-141. https://doi.org/10.1111/j.2044-8295.2011.02067.x

Daniel, T. O., Said, M., Stanton, C. M., \& Epstein, L. H. (2015). Episodic Future Thinking Reduces Delay Discounting and Energy Intake in Children. Eating Behaviors, 18, 20-24. https://doi.org/10.1016/j.eatbeh.2015.03.006

Daniel, T. O., Stanton, C. M., \& Epstein, L. H. (2013). The Future Is Now: Reducing Impulsivity and Energy Intake using Episodic Future Thinking. Psychological Science, 24, 2339-2342. https://doi.org/10.1177/0956797613488780

Dassen, F. C., Jansen, A., Nederkoorn, C., \& Houben, K. (2016). Focus on the Future: Episodic Future Thinking Reduces Discount Rate and Snacking. Appetite, 101, 229-230. https://doi.org/10.1016/j.appet.2016.02.102

Epstein, L. H., Salvy, S. J., Carr, K. A., Dearing, K. K., Bickel, W. K., Grill, H., \& Lowe, M. (2010). Food Reinforcement, Delay Discounting and Obesity. Physiology \& Behavior, 100, 438-445. https://doi.org/10.1016/j.physbeh.2010.04.029

Field, M., Christiansen, P., Cole, J., \& Goudie, A. (2007). Delay Discounting and the Alcohol Stroop in Heavy Drinking Adolescents. Addiction, 102, 579-586. https://doi.org/10.1111/j.1360-0443.2007.01743.x

Griffiths, A., Hill, R., Morgan, C., Rendell, P. G., Karimi, K., Wanagaratne, S., \& Curran, H. V. (2012). Prospective Memory and Future Event Simulation in Individuals with Alcohol Dependence. Addiction, 107, 1809-1816. https://doi.org/10.1111/j.1360-0443.2012.03941.x

Hall, P. A., \& Fong, G. T. (2007). Temporal Self-Regulation Theory: A Model for Individual Health Behavior. Health Psychology Review, 1, 6-52. https://doi.org/10.1080/17437190701492437

Hassabis, D., Kumaran, D., Vann, S. D., \& Maguire, E. A. (2007). Patients with Hippocampal Amnesia Cannot Imagine New Experiences. Proceedings of the National Academy of Sciences of the United States of America, 104, 1726-1731. 
https://doi.org/10.1073/pnas.0610561104

Hendrickson, K. L., \& Rasmussen, E. B. (2013). Effects of Mindful Eating Training on Delay and Probability Discounting for Food and Money in Obese and Healthy-Weight Individuals. Behaviour Research \& Therapy, 51, 399-409. https://doi.org/10.1016/j.brat.2013.04.002

Hershfield, H. E. (2011). Future Self-Continuity: How Conceptions of the Future Self Transform Intertemporal Choice. Annals of the New York Academy of Sciences, 1235, 30-43. https://doi.org/10.1111/j.1749-6632.2011.06201.x

Hill, E. M., Jenkins, J., \& Farmer, L. (2008). Family Unpredictability, Future Discounting, and Risk Taking. The Journal of Socio-Economics, 37, 1381-1396.

https://doi.org/10.1016/j.socec.2006.12.081

Johnson, A., \& Redish, A. D. (2007). Neural Ensembles in CA3 Transiently Encode Paths forward of the Animal at a Decision Point. Journal of Neuroscience the Official Journal of the Society for Neuroscience, 27, 12176-12189. https://doi.org/10.1523/JNEUROSCI.3761-07.2007

Kwan, D., Carson NAddis, D. R., \& Rosenbaum, R. S. (2010). Deficits in Past Remembering Extend to Future Imagining in a Case of Developmental Amnesia. Neuropsychologia, 48, 3179-3186. https://doi.org/10.1016/j.neuropsychologia.2010.06.011

Kwan, D., Craver, C. F., Green, L., Myerson, J., \& Rosenbaum, R. S. (2013). Dissociations in Future Thinking Following Hippocampal Damage: Evidence from Discounting and Time Perspective in Episodic Amnesia. Journal of Experimental Psychology General, 142, 1355-1369. https://doi.org/10.1037/a0034001

Kwan, D., Craver, C. F., Green, L., Myerson, J., Boyer, P., \& Rosenbaum, R. S. (2012). Future Decision-Making without Episodic Mental Time Travel. Hippocampus, 22, 1215-1219. https://doi.org/10.1002/hipo.20981

Liberman, N., \& Trope, Y. (2003). Construal Level Theory of Intertemporal Judgment and Decision. In D. Read, G. Loewenstein, \& R. Baumeister (Eds.), Time and Decision: Economic and Psychological Perspectives on Intertemporal Choice (pp. 245-276). New York: Russell Sage Foundation.

Liu, L., Feng, T., Chen, J., \& Li, H. (2013). The Value of Emotion: How Does Episodic Prospection Modulate Delay Discounting? PLoS ONE, 8, e81717.

https://doi.org/10.1371/journal.pone.0081717

Mackillop, J., Jr., M. R., Monti, P. M., Ray, L. A., Murphy, J. G., Rohsenow, D. J., Gwaltney, C. J. et al. (2010). Alcohol Demand, Delayed Reward Discounting, and Craving in Relation to Drinking and Alcohol Use Disorders. Journal of Abnormal Psychology, 119, 106-114. https://doi.org/10.1037/a0017513

Mariano, T. Y., Bannerman, D. M., Mchugh, S. B., Preston, T. J., Rudebeck, P. H., Rudebeck, S. R., Baxter, M. G. et al. (2009). Impulsive Choice in Hippocampal But Not Orbitofrontal Cortex-Lesioned Rats on a Nonspatial Decision-Making Maze Task. European Journal of Neuroscience, 30, 472-484.

Miedl, S. F., Peters, J., \& Büchel, C. (2012). Altered Neural Reward Representations in Pathological Gamblers Revealed by Delay and Probability Discounting. Archives of General Psychiatry, 69, 177-186. https://doi.org/10.1001/archgenpsychiatry.2011.1552

Noël, X., Saeremans, M., Kornreich, C., Jaafari, N., \& D’Argembeau, A. (2017). Future-Oriented Mental Time Travel in Individuals with Disordered Gambling. Consciousness \& Cognition, 49, 227-236. https://doi.org/10.1016/j.concog.2017.02.006

Odlaug, B. L., Schreiber, L. R., \& Grant, J. E. (2013). Personality Dimensions and Disorders in Pathological Gambling. Current Opinion in Psychiatry, 26, 107-112. 
https://doi.org/10.1097/YCO.0b013e32835997df

Palombo, D. J., Keane, M. M., \& Verfaellie, M. (2015). The Medial Temporal Lobes Are Critical for Reward-Based Decision Making under Conditions That Promote Episodic Future Thinking. Hippocampus, 25, 345-353. https://doi.org/10.1002/hipo.22376

Pepper, G. V., \& Nettle, D. (2013). Death and the Time of Your Life: Experiences of Close Bereavement Are Associated with Steeper Financial Future Discounting and Earlier Reproduction. Evolution \& Human Behavior, 34, 433-439. https://doi.org/10.1016/j.evolhumbehav.2013.08.004

Pepper, G. V., \& Nettle, D. (2014). Perceived Extrinsic Mortality Risk and Reported Effort in Looking after Health. Human Nature, 25, 378-392. https://doi.org/10.1007/s12110-014-9204-5

Peters, J., \& Büchel, C. (2010). Episodic Future Thinking Reduces Reward Delay Discounting through an Enhancement of Prefrontal-Mediotemporal Interactions. Neuron, 66, 138-148. https://doi.org/10.1016/j.neuron.2010.03.026

Peters, J., \& Büchel, C. (2011). The Neural Mechanisms of Inter-Temporal Decision-Making: Understanding Variability. Trends in Cognitive Sciences, 15, 227-239. https://doi.org/10.1016/j.tics.2011.03.002

Potenza, M. N. (2003). An fMRI Stroop Task Study of Ventromedial Prefrontal Cortical Function in Pathological Gamblers. The American Journal of Psychiatry, 160, 1990-1994. https://doi.org/10.1176/appi.ajp.160.11.1990

Schacter, D. L., \& Addis, D. R. (2009). On the Nature of Medial Temporal Lobe Contributions to the Constructive Simulation of Future Events. Philosophical Transactions of the Royal Society B Biological Sciences, 364, 1245-1253. https://doi.org/10.1098/rstb.2008.0308

Schacter, D. L., Addis, D. R., \& Buckner, R. L. (2007). Remembering the Past to Imagine the Future: The Prospective Brain. Nature Reviews Neuroscience, 8, 657-661.

https://doi.org/10.1038/nrn2213

Scherbaum, S., Haber, P., Morley, K., Underhill, D., \& Moustafa, A. A. (2017). Biased and Less Sensitive: A Gamified Approach to Delay Discounting in Heroin Addiction. Journal of Clinical \& Experimental Neuropsychology, 40, 139-150.

Snider, S. E., Laconte, S. M., \& Bickel, W. K. (2016). Episodic Future Thinking: Expansion of the Temporal Window in Individuals with Alcohol Dependence. Alcoholism-Clinical \& Experimental Research, 40, 1558-1566.

https://doi.org/10.1111/acer.13112

Stein, J. S., Renda, C. R., Hinnenkamp, J. E., \& Madden, G. J. (2015). Impulsive Choice, Alcohol Consumption, and Pre-Exposure to Delayed Rewards: II. Potential Mechanisms. Journal of the Experimental Analysis of Behavior, 103, 33-49. https://doi.org/10.1002/jeab.116

Stein, J. S., Wilson, A. G., Koffarnus, M. N., Daniel, T. O., Epstein, L. H., \& Bickel, W. K. (2016). Unstuck in Time: Episodic Future Thinking Reduces Delay Discounting and Cigarette Smoking. Psychopharmacology, 156, 1-8. https://doi.org/10.1007/s00213-016-4410-y

Stevens, J. R., \& Stephens, D. W. (2008). Patience. Current Biology, 18, R11-R12. https://doi.org/10.1016/j.cub.2007.11.021

Suddendorf, T., \& Corballis, M. C. (2010). Behavioural Evidence for Mental Time Travel in Nonhuman Animals. Behavioural Brain Research, 215, 292-298. https://doi.org/10.1016/j.bbr.2009.11.044

Suddendorf, T., \& Moore, C. (2011). Introduction to the Special Issue: The Development of Episodic Foresight. Cognitive Development, 26, 295-298. 
https://doi.org/10.1016/j.cogdev.2011.09.001

Trope, Y., \& Liberman, N. (2000). Temporal Construal and Time-Dependent Changes in Preference. Journal of Personality and Social Psychology, 79, 876-889. https://doi.org/10.1037/0022-3514.79.6.876

Vanderbroek-Stice, L., Stojek, M. K., Beach, S. R., Vandellen, M. R., \& Mackillop, J. (2017). Multidimensional Assessment of Impulsivity in Relation to Obesity and Food Addiction. Appetite, 112, 59-68. https://doi.org/10.1016/j.appet.2017.01.009

Vuchinich, R. E., \& Heather, N. (2003). Choice, Behavioural Economics and Addiction (pp. 427-432). New York: Elsevier.

Wang, Q., \& Zhang, E. (2015). Methods for Reducing Impulsiveness in Decision-Making and Its Neural Mechanisms. Advances in Psychological Science, 23, 101-109. https://doi.org/10.3724/SP.J.1042.2015.00101

Wiehler, A., Bromberg, U., \& Peters, J. (2015). The Role of Prospection in Steep Temporal Reward Discounting in Gambling Addiction. Frontiers in Psychiatry, 6, 700-706.

Wu, W. H., Cheng, W., \& Chiou, W. B. (2017). Episodic Future Thinking about the Ideal Self Induces Lower Discounting, Leading to a Decreased Tendency toward Cheating. Frontiers in Psychology, 8, 287. https://doi.org/10.3389/fpsyg.2017.00287

Yi, R., Mitchell, S. H., \& Bickel, W. K. (2010). Delay Discounting and Substance Abuse-Dependence. In G. J. Madden, \& W. K. Bickel (Eds.), Impulsivity: The Behavioral and Neurological Science of Discounting (pp. 191-211). Washington DC: American Psychological Association. https://doi.org/10.1037/12069-007 